\title{
La leadership educativa nel sistema scolastico italiano: tra istituzioni e competenze
}

\section{Titti Romano e Roberto Serpieri}

Il lavoro è frutto di studi e riflessioni comuni, ma è da intendere che la stesura dei paragrafi è personale e indicato con la rispettiva sigla.

L'obiettivo di questo articolo è fornire una lettura della leadership educativa attraverso una prospettiva che permetta di vedere la leadership come una "competenza emergente» (duratura, autonoma ed indipendente), esito della complessa relazione tra individuo e contesto (Archer). Il leader educativo non è nient'altro che un soggetto competente capace di monitorare riflessivamente (conversazioni interne) sé stesso (desideri, passioni, progetti) e i vincoli e le risorse istituzionali. La chiave di lettura proposta sarà applicata per l'analisi di uno studio di caso: da un lato, un contesto istituzionale (il sistema scolastico italiano) travolto da un processo di riforma che sollecita $i$ leader all'assunzione di nuove competenze (dirigenza scolastica), dall'altro, un leader educativo con una lunga carriera professionale alle spalle. Le competenze di leadership emergeranno da questa complessa relazione tra elementi che si condizionano senza né fondersi (ontologia della prassi) né determinarsi (riduzionismo), in quanto irriducibili (secondo il realismo critico archeriano).

\section{Dalla "leadership istituzionale» alle "competenze emergenti» di leadership (RS)}

L'obiettivo di questo articolo è quello di presentare una lettura dei processi di costituzione della leadership della dirigenza scolastica, con particolare riferimento al caso del sistema educativo italiano ${ }^{1}$. In particolare, piuttosto che tentare di contribuire alla continua proliferazione di aggettivi accoppiati al termine leadership, si vuole offrire un'ipotesi per interpretare la costruzione di competenze di leadership. Considerare sul serio il termine "costruzione» permetterà così di analizzare le competenze di leadership nei termini di un processo che, nel tempo, porta gli attori sociali a ricoprire diversi ruoli, che a loro volta sono modellati da un insieme di condizionamenti struttural-culturali. Come si tenterà di 
argomentare, queste competenze saranno viste come proprietà «emergenti» (Archer, 1995), ovvero relativamente indipendenti dallo stesso individuo che le pratica e derivate, come esiti mai scontati e del tutto prevedibili, dalla storia della sua interpretazione di più ruoli in contesti modellati istituzionalmente.

Un saggio sulla leadership educativa non può prescindere dalla sconfinata letteratura in materia e, tuttavia, in questa sede non si potrà che procedere in modo sintetico nella ricostruzione del dibattito fin qui sviluppato. Il primo punto, quindi, da introdurre come premessa alla discussione è quello dell'evoluzione degli approcci al tema della leadership educativa e delle relative competenze, che ha portato ad evadere dalle concezioni centrate sul singolo attore per giungere, invece, ad una focalizzazione sulla dimensione processuale e collettiva. Il secondo aspetto rinvia, inoltre, ai processi di trasformazione dei sistemi educativi che hanno visto un progressivo scivolamento delle politiche, dei programmi e delle retoriche dall'educational leadership all'educational management nell'ultimo ventennio del secolo scorso, salvo ripensamenti e rivolgimenti che, a cavallo dei due milleni, stanno riportando in auge la prima a scapito del secondo ${ }^{2}$.

Per quanto riguarda l'evoluzione degli approcci al tema della leadership educativa, si può osservare che questa ha seguito nel complesso quella degli studi sulla leadeship intesa come fenomeno più generale, attraversando le classiche fasi: dalla focalizzazione sui tratti di personalità e sugli stili alla dialettica del leader «transazionale-trasformazionale»; passando per le fasi «situazioniste»e "contingentiste», per poi riprendere gli echi trasformazionalisti e culturalisti nei termini di una rivisitazione della dimensione carismatica weberiana (Bryman, 1992; Sims \& Lorenzi, 1992); per finire con le più recenti «critiche» (Gronn \& Ribbins, 1996), ispirate a correnti postmoderniste e postpositiviste, o con le visioni che hanno connesso sempre più la leadership a concezioni distributive e processuali (Gronn, 2000), nei termini, ad es., del contributo dato dalla leadership all'apprendimento organizzativo (Sadler, 2000). Nel suo complesso, tuttavia, si ritiene che sia possibile ricondurre la stragrande maggioranza degli approcci a due principali filoni interpretativi: quelli che, in qualche modo, è possibile fare risalire alle due componenti politica e simbolica della leadership istituzionale di Selznick (1957). Il «leader istituzionale», infatti, si attiva politicamente e simbolicamente per definire missione e ruolo istituzionali; incorporare la linea politica nella struttura sociale, ovvero formarne il «carattere»; difendere l'integrità istituzionale, cioè definire i valori comuni e l'identità distintiva; ricomporre, infine, i conflitti interni.

Nell'ambito, quindi, del filone simbolico della leadership educativa possono farsi rientrare quegli autori che hanno enfatizzato innanzitutto la componente cosiddetta morale della leadership (Hodgkinson, 1991; Sergiovanni, 1992). Ma l'approccio che si distingue come il più significativo in questo filone è senz'altro quello della cosiddetta leadership "trasformazionale» (Leithwood, Tomlinson \& Genge, 1996) impegnata nella gestione delle culture organizzative delle scuole per temi quali: il carisma, l'ispirazione e la vision; il consenso sui fini e la consi- 
derazione individuale; la stimolazione intellettuale ed il modellamento di comportamenti ed atteggiamenti; la costruzione della cultura della scuola. Quest'approccio, tuttavia, riprendendo temi quali la contingenza delle ricompense, le elevate prospettive di performance e la strutturazione dei ruoli e delle risorse, si presenta con sensibili margini di commistione con approcci di impronta manageriale ed in tal senso è stato oggetto di una puntuale disamina critica. In nome, infatti, di una "post-trasformational leadership» resistente ai canti delle sirene del new public management e più concorde invece con un atteggiamento neo-professionale, si evidenzia la sostanziale sottovalutazione di una serie di "qualità interpersonali e di capacità necessarie ed appropriate per una leadership efficace» (Day et al., 2000, p. 177), quali: riflessività e pensiero critico, introspezione sui valori, artistry e dimensioni emozionali ed, infine, focalizzazione sullo sviluppo professionale e gestione degli attori in conflitto nella knowledge society. Gli stessi principali esponenti della leadership trasformazionale, hanno rivisitato tale approccio nei termini di «quelle concezioni relativamente recenti della leadership come qualcosa di ampiamente distribuito attraverso le organizzazioni e [con] lo scopo centrale di tale leadership: l'empowerment degli altri» (Leithwood, Jantzi \& Steinbach, 1999, p. 4). Non vanno, infine, dimenticati quegli autori che hanno esteso le potenzialità interpretative insite nel filone simbolico nel riprendere le problematiche del confronto della leadership con le differenze di contesti e di culture (Dimmock \& Walker, 2005).

Nell'alveo del secondo filone si distingue, indubbiamente, l'approccio alle «micropolitiche» che gli attori pongono in essere (Bacharach \& Mundell, 1993, 1995), di cui Ball (1987) con la sua distinzione degli stili di leadership rappresenta un "classico». Con riferimento alla questione delle specificità organizzative delle scuole, alla loro natura di "gerarchie confuse» (Shedd \& Bacharach, 1991), non si può, poi, non ricordare l'importante contributo per la definizione delle caratteristiche di una leadership democratica offerta da Blase. Questi, incrociando le due dimensioni del grado di apertura o chiusura dello stile di leadership e della natura transattiva o trasformativa ${ }^{3}$ dei fini perseguiti dai leader scolastici (Blase \& Anderson, 1995), ha individuato quattro tipi di leadership. Tra questi va citata, in quanto anticipatrice rispetto agli sviluppi del filone politico, quella "democratica» per la sua capacità di empowerment sociale, di dialogo «autentico» ed emancipatorio e di con-divisione del potere («potere con»). Tra le ulteriori visioni di leadership che hanno enfatizzato, in chiave polemica con l'approccio trasformazionale, una precipua dimensione di politicità si segnala, ad es., quella della Telford, (1996). La sua proposta di leadership "collaborativa» rinvia alla necessità di considerare la dimensione "densa» del potere in favore di una distribuzione dello stesso agli altri «committed-professionals», in primo luogo gli insegnanti.

Un'altra recente proposta in questa direzione è la «sustainable leadership» con i suoi «sette principi» (Hargreaves \& Fink, 2003), molto in sintonia con le prospettive distributive e con le letture in termini di promozione da parte della lea- 
dership dello sviluppo delle (comunità di) pratiche professionali nelle scuole. Tale proposta, tuttavia, rimane legata ad una concezione della leadership sostanzialmente individualista e, pur ponendosi nell'ottica di un rilancio del professionalismo educativo, rimane alquanto ambigua rispetto ai processi sociali che sosterrebbero l'affermarsi di una siffatta leadership. Nella descrizione dei casi che viene offerta dai suoi proponenti, infatti, non si comprende se la chiave di volta continui ad essere rappresentata dall'azione del singolo leader e/o dalla forza del contesto: quando, ad es., si narra del caso di una resistenza ai processi di standardizzazione imposti in una scuola, non risulta chiara la fonte di una tale resistenza. In primo luogo, infatti, non c'è alcun accenno alla natura delle pressioni istituzionali in termini di condizionamenti dell'azione; nè si comprende se la storia delle competenze del leader esibisca una sua coerenza contro la cultura valutativa della standardizzazione, nè tantomeno si comprende se il contesto, gli stessi insegnanti, agiscano come un attore collettivo dove la leadership appaia come più o meno distribuita ed in virtù di quale fattore di influenza. Insomma, il quadro fornito della leadership «sostenibile», nonostante le sollecitazioni offerte alla riflessione sulla natura del fenomeno leadership educativa in epoche di profonde trasformazioni, continua ad essere sguarnito di un quadro interpretativo all'altezza della complessità della fenomenologia indagata. La normatività del modello, tratto questo che l'accomuna ai vari modelli di leadership aggettivata, viene peraltro confermato dagli inviti, di chiaro sapore prescrittivo-managerialista, ad un'adozione generalizzata dei suddetti «sette principi» per la sostenibilità della leadership.

Anche la "democratic leadership» (Woods, 2005), infine, che ha ultimamente ripreso gli spunti emancipativi presenti nella stragrande maggioranza degli approcci risalenti al filone politico, tende a riprodurre una visione normativa. Al di là dell'indubbio interesse che offre questa tensione, per quanto prescrittiva, verso una democraticità delle istituzioni scolastiche, il modello si segnala comunque per alcuni spunti interpretativi molto prossimi alla proposta che si presenta in quest'articolo. Woods, infatti, ispirandosi alla Archer $(1995,2000)$, ritiene che per spiegare le dinamiche sociali debbano prendersi in considerazione i tre elementi che compongono il suo modello «trialettico». La «struttura», innanzitutto, che attraverso alcune "proprietà» istituzionali, culturali e sociali rappresenta il contesto organizzativo dato per le mosse degli attori sociali. Questi ultimi, definiti più propriamente come "persone», si confrontano con i condizionamenti strutturali potendo contare sulle proprie capacità ed abilità. Il terzo elemento è costituito dall' «engagement», ovvero da come le persone interpretano ed agiscono e di qui la natura interattiva del rapporto tra struttura e persone. "Attraverso il loro engagement - sostiene, infatti, Woods - le persone modellano le stesse strutture organizzative che le abilitano e che le vincolano" (ivi, p. xviii). Sono, quindi, contenuti in nuce alcuni presupposti irrinunciabili per interpretare non solo come un contesto di leadership debba o non debba rispondere alle caratteristiche di questo o quel modello. Questi presupposti interpretativi, piuttosto, potreb- 
bero permettere di fondare su dati di ricerca come possano emergere contesti, pratiche e competenze di leadership educativa più o meno democratica, piuttosto che distribuita o sostenibile, ecc. Tali presupposti sono essenzialmente due: una specifica concezione dell'agency ed una prospettiva diacronica. Non si può non notare, tuttavia, come questi presupposti finiscano col restare appena enunciati; nel senso che il modello "trialettico" sembrerebbe confinato in una, peraltro blanda, prospettiva di strutturazione (vedi l'analogia rispettivamente delle "persone» e dell" «engagement» con gli "attori competenti» e con la "condotta strategica» di Giddens, 1984), al di là del richiamo alla Archer che, come noto, ha profondamente criticato tale prospettiva. Ma, soprattutto, nel senso che la preoccupazione principale di Woods sembra risiedere più nella definizione degli elementi distintivi del modello, piuttosto che nella messa alla prova dei presupposti interpretativi per testimoniare attraverso ricerche come si possa giungere alla messa in pratica di una leadership democratica. E ciò appare tanto più grave quanto più si consideri in quale misura la leadership educativa venga senpre più sottoposta alle pressioni ed ai condizionamenti del public management (Ball, 1994, 2006), che, certamente, non risulta particolarmente sintonico con le tensioni emancipatorie che contraddistinguono il filone politico degli approcci di leadership.

Non è, quindi, un caso che, con sempre più enfasi a partire dagli anni ottanta, si vada insistendo su politiche di costruzione e circolazione di vere e proprie "coorti» di leader informati da opzioni valoriali ben definite (Gronn \& Ribbins, 1996), sempre più schiacciate dalla dialettica tra due opposte prospettive: quella empatica dell' "insightful educational leadership» e quella razionalista dell'educational management (Leithwood, Tomlinson \& Genge, 1996; Levacic, Glover, Bennett, \& Crawford, 1999). Quest'ultima si è, infatti, progressivamente affermata anche nel mondo della scuola, in sintonia con la retorica del public management (Battistelli, 2002) che viene presentato come una potente leva per l'ottenimento di livelli più elevati di efficienza ed efficacia nei sistemi pubblici. L'ideologia del "dominio dei manager» che sottende la svolta decisionista (Helsby, 1999) ha finito, quindi, per contraddistinguere tipicamente la logica dell' "amministrazione imprenditoriale» (D'Albergo \& Vaselli, 1999), a discapito di logiche ispirate da una più convinta fiducia nei metodi democratici. In tale scenario, pur contraddistinto dalle diversità dei vari sistemi educativi, sembrerebbe che sulle spalle del dirigente scolastico vengano a ricadere le più disparate e spesso confliggenti richieste di accountability (Barzanò, in stampa).

Prendendo, quindi, in considerazione le conseguenze delle stagioni di riforma dei sistemi educativi occidentali per le figure dei dirigenti scolastici, si potrebbe notare come da più parti si cerchi di stabilire quali nuove competenze siano necessarie affinché si possa essere/diventare dirigenti scolastici di «successo». Una vera e propria "febbre» da repertori di competenze sembra, quindi, diffondersi in modo pervasivo in riferimento ai vari processi di selezione, valutazione, formazione, ecc., dei leader educativi, così come aveva già investito la professione do- 
cente e soprattutto la valutazione degli apprendimenti dei discenti (Benadusi, 2002). Tali repertori, attraverso l'elenco delle caratteristiche della personalità e delle prestazioni emblematiche, "confezionano" un leader capace di operare con successo in qualsiasi tipo di istituzione educativa. Il leader educativo sembra divenire un elemento strategico che incide profondamente sulle performance di quanti, professori o alunni, si trovano ad operare nel suo istituto, in altre parole, il leader rappresenta quel fattore che determina il successo o l'insuccesso dell'istituto scolastico.

La proposta interpretativa che, invece, viene presentata nel prossimo paragrafo intende:

- tanto minare la credenza in un leader eroicamente concepito come un paladino della resistenza dei valori di una scuola "pura», quasi una sorta di isola moralmente autosufficiente rispetto alle pressioni dell'ambiente istituzionale;

- quanto ribadire una netta presa di distanza da quelle visioni delle competenze del dirigente scolastico, intese quali asettico passe par tout per una gestione della scuola secondo principi manageriali di per sé «neutrali».

\section{Le “competenze emergenti» de $i$ leader educativi italiani (TR)}

In questo paragrafo svilupperemo in dettaglio la nostra proposta interpretativa e procederemo ad una sua prima applicazione analizzando l'agire professionale dei neo dirigenti italiani attraverso il concetto di "competenza emergente». Lo sviluppo delle competenze ${ }^{4}$, inteso come un processo né esclusivamente sociale, né esclusivamente individuale, sarà analizzato attraverso il «dualismo analitico»su cui si basa la proposta teorica di Archer (1995, 2000, 2003): una prospettiva stratificata che riconosce da un lato, l'importanza delle influenze sociali (condizionamenti struttural-culturali) che impattano sui contesti di interazione e contribuiscono a plasmare e modellare i ruoli e, dall'altro, la relativa autonomia delle proprietà personali degli individui (identità personale e sociale) che tali ruoli interpretano ${ }^{5}$. Il concetto di competenza è, quindi, riconcettualizzato come elemento di sintesi che emerge dalla relazione tra attore e struttura, consentendoci di analizzare la figura dei leader educativi italiani senza cadere in letture riduzionistiche dell'agire (Romano \& Serpieri, 2003, 2004, 2006). Letture, cioè, legate a concezioni sovra-socializzate, che vedono l'individuo come un passivo prodotto delle strutture socio-culturali o letture sotto-socializzate in cui, di converso, gli individui sono liberi artefici del destino proprio e della società.

In estrema sintesi, l'ipotesi da cui partiamo è che l'interpretazione di un ruolo è strettamente correlata alle competenze che l'individuo acquisisce per far fronte agli impegni professionali. Tali competenze sono frutto di una tensione tra individuo e società. Le competenze, infatti, sono connesse a caratteristiche profonde e durature della personalità degli individui e quindi sono elementi persistenti 
non soggetti a cambiamenti repentini. Il loro manifestarsi, però, è connesso all'esercizio di una specifica attività in un determinato contesto; è, quindi, influenzato anche dalle modalità con cui è possibile svolgere un lavoro (quote di autonomia e responsabilità, struttura gerarchica e d'autorità, ripartizione delle attività, margini di innovazione, ecc).

Le competenze si costruiscono, attraverso un processo a spirale che costituisce la metafora del percorso e delle tensioni tra l'identità personale del dirigente scolastico ed i condizionamenti esterni all'azione. La separazione è naturalmente solo analitica poiché c'è «un'unica storia da raccontare» in cui le condotte dei dirigenti, vanno intese come esito scaturente dalle riflessioni («conversazioni interiori» - Archer, 2003) che, in quanto persone datate di una personalità individuale, effettueranno per filtrare, valutare gli esiti delle loro azioni e la realtà del contesto in cui operano. Come è rappresentato nella figura 1 c'è, quindi, una persona attiva e riflessiva con una sua identità personale che interpreta i numerosi ruoli sociali, acquisisce competenze, filtra e valuta le esperienze lavorative durante tutto il percorso che caratterizzerà la sua carriera.

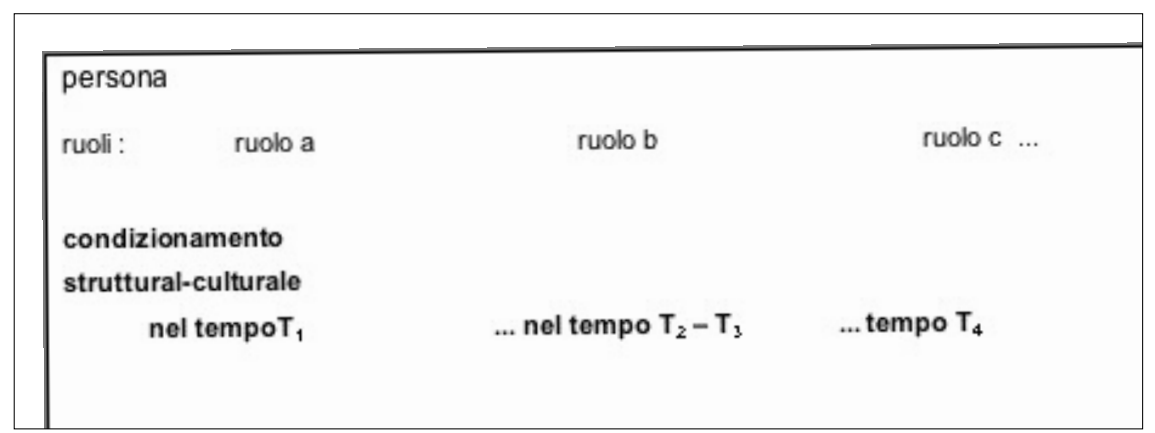

Fig. 1. La spirale dello sviluppo delle competenze individuali

Nota fig. 1: le scansioni temporali $\left(\mathrm{T}_{1}, \mathrm{~T}_{2}-\mathrm{T}_{3}\right.$ e $\left.\mathrm{T}_{4}\right)$ sono inserite per evidenziare i macro cambiamenti a livello di sistema che condizionano lo sviluppo delle competenze individuali (cfr. fig. successive).

Il set di competenze che si acquisisce in tale dinamica può essere considerato come una "proprietà emergente» degli individui in quanto, nel corso degli anni, assume lo status di elemento preesistente e duraturo che contraddistingue il leader educativo. In tale dinamica, la spirale diviene un elemento chiave poiché solo con il trascorrere del tempo le competenze possono configurarsi quali "proprietà emergenti» che condizionano i futuri corsi d'azione, ovvero, acquisiscono una durata temporale che le rendono relativamente indipendenti dalle altre dimensioni rilevanti, non ultimi anche i desideri degli stessi attori. La metafora della spirale ci permette di dare alla dimensione temporale una nuova centralità e di evidenziare come il processo dialettico si sviluppa lungo tutto l'arco dell'esistenza 
di un individuo: le scelte di oggi possono essere comprese solo alla luce di quelle di ieri.

Una ricerca qualitativa: Lo studio di un caso (TR)

La riflessione teorica appena esposta ha dato, luogo ad un confronto con la realtà empirica attraverso uno studio di caso longitudinale che riteniamo, per una prima ricerca esplorativa, la metodologia più efficace per osservare un fenomeno che si dipana lungo un arco temporale piuttosto esteso. La ricerca si compone di due parti: un'analisi dell'evoluzione del sistema scolastico italiano e un vero e proprio studio di caso longitudinale. Prima di affrontare lo studio di caso è stato necessario, dare una breve panoramica dei "condizionamenti cultural-strutturali». In definitiva:

- nella prima parte analizzeremo l'evoluzione dei «condizionamenti culturalstrutturali» che caratterizzano il sistema scolastico italiano tuttora al centro di una riforma che, dal 1997, ha inaugurato una fase di profonda trasformazione. L'obiettivo è comprendere i cambiamenti culturali e strutturali che dall'esterno impattano sui vari contesti di interazione creando nuovi ruoli e responsabilità. In particolare, i leader educativi transitano da un ruolo prevalentemente burocratico ed amministrativo (direttori didattici e presidi), all'attuale ruolo di dirigenti, caratterizzato da più ampia autonomia e responsabilità;

- svilupperemo poi, nella seconda parte, uno studio di caso longitudinale per cogliere, a livello di interazione sociale, il processo di sviluppo delle "competenze emergenti» individuali che sarà rappresentato attraverso una spirale.

Per cogliere le principali caratteristiche del processo di riforma (Benadusi, 1989; Ventura, 1998) che contraddistingue il sistema scolastico italiano si utilizzerà una sintetica stilizzazione del modello archeriano del ciclo «morfogenetico» che prevede tre fasi: la fase del condizionamento struttural-culturale, la fase dell'interazione sociale e la fase del nuovo assetto struttural-culturale (cf. Fig. 2).

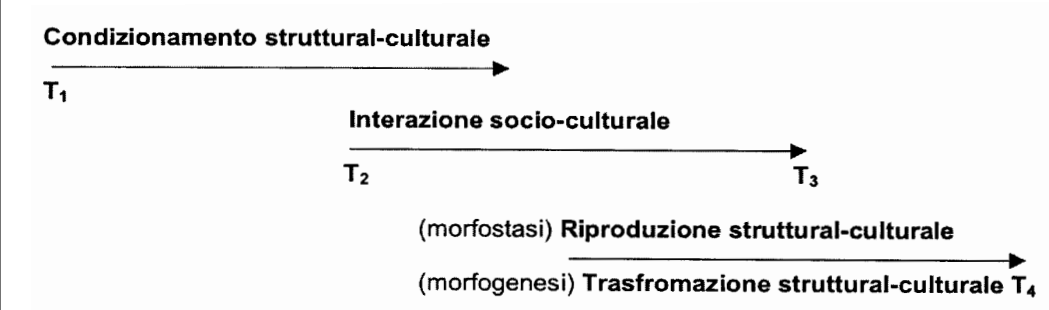

Fig. 2. Ciclo «morfogenetico/morfostatico» Fonte: riadattato da Archer, 1995 
Secondo tale modello i condizionamenti struttural-culturali, che sono il risultato dell'interazione umana passata, precedono temporalmente la fase dell'interazione sociale, che rappresenta il momento in cui gli agenti riproducono (morfostasi) o trasformano (morfogenesi) il sistema (nel caso italiano assistiamo ad un tentativo di morfogenesi ancora in fieri); la terza ed ultima fase è caratterizzata dall'elaborazione o riproduzione struttural-culturale.

In riferimento allo studio di caso, si è partiti dal presupposto che «il campionamento nella ricerca qualitativa non è né statistico né puramente personale: è, o dovrebbe essere, teoricamente fondato» (Silverman, 2000, p. 159). Il caso è stato selezionato, quindi, secondo i criteri del campionamento a scelta ragionata - theoretical sampling - considerando sia la rilevanza che esso ha per le ipotesi di ricerca, sia la possibilità di ritrovare caratteristiche o dimensioni che ci aiutano a sviluppare a controllare tale ipotesi. In particolare, abbiamo optato per un caso deviante (ibidem), ovvero un caso che risulta in contraddizione sia rispetto alla teoria utilizzata, sia rispetto alla situazione tipica della realtà italiana (Fisher, Fisher \&Masuelli, 2002). Il leader educativo dai noi selezionato si configura, per molti versi, come un caso anomalo rispetto al panorama italiano e sembrerebbe confermare un'ipotesi individualista (letture sotto-socializzate). Si è ritenuto un buon banco di prova affrontare questo studio di caso così apparentemente contrastante con le nostre ipotesi. Il caso è stato selezionato tra quelli già effettuati nel 1998 all'interno di una ricerca più ampia condotta su scala nazionale. Gli studi di caso condotti, infatti, avevano rintracciato alcuni presidi e insegnanti che, attraversando una pluralità di esperienze, viverano ed interpretarano i loro ruolo in modo proattivo e creativo, acquisendo competenze che permettessero loro di uscire dalle prescrizioni di un ruolo prevalentemente burocratico ed amministrativo (Serpieri, 1999). Si è portato a termine, quindi, uno studio longitudinale che, rintracciando gli avvenimenti antecedenti l'autonomia, fosse in grado di cogliere il momento di svolta del sistema scolastico italiano (la ricerca è stata, infatti, ripresa in due successive fasi: nell'anno scolastico 2000/2001 e successivo)

Come è tradizione degli studi di caso, anche nella nostra ricerca sono state utilizzate una pluralità di tecniche ed in particolare:

- osservazioni del dirigente, collegio dei docenti, consiglio di istituto, riunioni del preside con il team delle f.o. (funzioni obiettivo) e dei collaboratori;

- Bei - Behavioural Event Interview ${ }^{6}$ al dirigente e alle figure di staff;

- interviste in profondità al preside, collaboratori e alle figure di staff;

- analisi di alcuni documenti della scuola: POF (Piano dell'Offerta Formativa), relazioni conclusive delle figure di staff, accordi di rete, ecc.

Per rilevare lo sviluppo delle «competenze emergenti» è stato necessario integrare la tecnica dell'osservazioni con le Bei; la prima per cogliere direttamente gli aspetti comportamentali e situazionali e la seconda per comprendere dimensioni non osservabili come gli schemi cognitivi, le intenzioni e le motivazioni che hanno guidato l'intervistato. Le "conversazioni interiori» (Archer, 2003) dei 
protagonisti sono state ricostruite sia attraverso le interviste in profondità e le Bei sia discutendo gli esiti di tali interviste direttamente con il protagonista. Per quanto riguarda la rilevazione dei condizionamenti esterni ci siamo soffermati prevalentemente su quelli presenti a livello di istituto ed, in particolare, su quelli esercitati dagli insegnanti (attivismo, dissenso, indifferenza, immobilismo) sulle attività del preside. Come vedremo, alcuni di questi condizionamenti, che impattano sull' esercizio e lo sviluppo delle competenze del preside, risentono fortemente dell'evoluzione del sistema scolastico nazionale (rilevati ed analizzati sinteticamente nel paragrafo successivo); altri condizionamenti, sono espressione, invece, del contesto locale (come ad esempio la possibilità di accedere ai consistenti finanziamenti europei, visto che la Campania resta ancora tra quelle Regioni che godono dello statuto di «obiettivo 1»). L'osservazione dei consigli e dei collegi dei docenti, integrata dall'analisi dei documenti ufficiali dell'istituto e dalle dichiarazioni degli intervistati, è stata la tecnica di indagine privilegiata per individuare tali condizionamenti. I dati raccolti sono stati analizzati attraverso un'analisi interpretativa e, in riferimento alla ricostruzione delle "conversazioni interiori» del leader, si è utilizzato un approccio narrativo (Silverman, 2002). In quest'ultimo caso il principale interesse era, infatti, comprendere la prospettiva del protagonista, come egli raccontava le sue esperienze e come tali narrazioni e riflessioni costituivano elementi significativi che incidono sulle sue azioni.

\section{Il ciclo "morfogenetico" del sistema educativo italiano (RS)}

L'evoluzione del sistema educativo italiano, qui presentata, è estremamente sintetica poiché ha solo l'obiettivo di rintracciare i principali condizionamenti che possono incidere sui contesti di interazione, contribuendo in modo più o meno diretto (erogazione di risorse, definizione dei ruoli, controllo dei processi decisionali, percorsi di attribuzione di senso, ecc,) alla formazione delle «competenze emergenti» dei leader educativi. Tali condizionamenti, però, come vedremo non determinano l'azione dei leader, la quale resta libera pur subendo, in positivo o in negativo, $\mathrm{i}$ costi ed $\mathrm{i}$ benefici dettati dal posizionamento nella struttura (cfr. Fig. 3).

Ci limiteremo ad analizzare brevemente i principali condizionamenti che influenzano i ruoli che la normativa italiana, in due periodi distinti, attribuisce ai leader educativi:

- il primo periodo $\left(T_{1}\right)$, che si è definito della pre-autonomia, si riferisce ad una lunga fase in cui i condizionamenti strutturali e culturali «modellano l'ambiente sociale»; in questa fase i leader educativi italiani occupano i ruoli di direttrici didattiche, di presidi e di capi d'istituto;

- la fase attuale $\left(\mathrm{T}_{2}-\mathrm{T}_{3}\right)$, dove la riforma dell'Autonomia del sistema scolastico (iniziata nel 1997) sta conoscendo la sua prima implementazione ed alle direttrici didattiche ed ai capi d'istituto viene attribuito il nuovo ruolo di dirigente scolastico. 


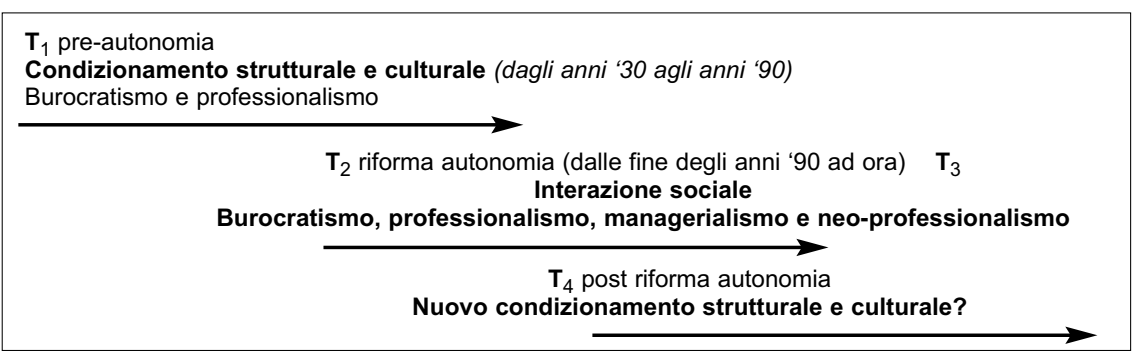

Fig. 3. Evoluzione del sistema educativo in Italia Fonte: riadattato da Archer, 1995

\section{Il tempo T1: la pre-autonomia}

Nel tempo $T_{1}$, abbiamo individuato i due maggiori vettori che veicolano i condizionamenti cultural-strutturale del sistema italiano configurando la scuola italiana come un tipico caso di «burocrazia professionale» (Mintzberg, 1985; Benadusi \& Serpieri, 2000b):

- il condizionamento al «burocratismo», consiste nella logica del «rendere conto» in base a criteri di evidenza formale e garantisti; ci si trova, infatti, in una situazione di condizionamento che presenta una forte accentuazione centralistico-burocratica, dove lo Stato mantiene il controllo pressocchè esclusivo di tutto il comparto educativo (Biggiero, 2000), spesso innescando dinamiche perverse di inefficienza, spreco e stasi (Ribolzi, 1997);

- il secondo condizionamento, più sfumato, definito "professionalismo", favorisce l'espressione di un portato di conoscenze, di pratiche e di valori rispondenti ad una matrice di formazione essenzialmente psico-pedagogica da un lato (così è stato soprattutto nel caso dei circoli didattici), e/o di tipo disciplinare da un altro lato (come nel caso soprattutto delle scuole medie inferiori e superiori). Le problematiche che sorgono da questo tipo di condizionamento incidono in modo riflesso sui dirigenti, influenzando più direttamente le logiche d'azione dei docenti: isolamento professionale, scarsa partecipazione alle attività collegiali soprattutto per quelle rivolte all'innovazione e la sperimentazione della didattica), nessun interesse per le questioni organizzative e gestionali della scuola.

In questa fase, i leader non erano sollecitati ad esprimere strategie personali nell'interpretazione autonoma del ruolo, ma erano semmai richiamati all'aderenza alle prescrizioni formali.

\section{Il tempo $T_{2-} T_{3}$ : la riforma}

La questione appare alquanto differente sul finire degli anni '80, quando il sistema scolastico italiano, anche se in ritardo e con una certa lentezza, si trova ad affrontare la profonda crisi che aveva colpito e trasformato i vari sistemi di welfare occidentali già dagli anni ' 70 . All'interno del dibattito la figura dei leader si trova al centro della diatriba prevalentemente ideologica che si interroga sul 
nuovo ruolo del leader educativo: «maestro dei maestri» o "preside manager» (Fisher, et al., 2002). Dibattito che anticipa dilemmi e perplessità che accompagnano tutta la fase di transizione che si inaugura con la Riforma dell'Autonomia Scolastica (L. 59/97, L. 53/03). È entro questo ampio processo di riforma che nasce la figura del dirigente scolastico, la quale sostituisce quelle dei direttori didattici e dei presidi. Il consistente aumento di retribuzione lo testimonia (sia rispetto al precedente ruolo, sia in termini di differenziazione dalla categoria degli insegnanti) e la formalizzazione di nuovi percorsi di selezione/reclutamento e di valutazione dei risultati.

In questa fase di transizione, si sviluppano spinte contraddittorie con esiti cumulativi e non si proviene ancora ad un nuovo assetto sistemico con precisi condizionamenti:

- si diffonde, infatti, un nuovo condizionamento in favore delle pratiche e delle retoriche del new public management (Battistelli, 2002), da noi definita «managerialismo», che si accompagna ad un'accentuazione delle attività di valutazione, monitoraggio, ecc., finalizzate al migliorare della "qualità» di una scuola vista soprattutto in un'ottica concorrenziale e, quindi, orientata non solo all'efficienza, ma soprattutto alla customer satisfaction;

- come è avvenuto negli altri sistemi educativi occidentali, si inizia ad inserire progressivamente un altro tipo di condizionamento che si definisce «neo-professionalismo» 7 , che, reclamando la specificità delle organizzazioni scolastiche e la inapplicabilità del discorso manageriale, rilanciano le logiche professionali, ma in una prospettiva collegial-comunitaria opposta a quella individualista;

- si può osservare come il condizionamento dominante nella fase pre-autonomistica, definito «burocratismo», sembri solo apparentemente eclissarsi (Benadusi \& Landri, 2002), ispirando ancora nei neo dirigenti quello stile rassicurante largamente informato allo spirito legalistico-formale;

- anche il condizionamento al "professionalismo" sopravvive e non scompare affatto di scena.

Sintetizzando l'impatto che la riforma sta operando sui leader scolastici, si può osservare che questa è tipicamente una fase di transizione dagli esiti poco scontati in cui si sovrappongono differenti ed anche opposte logiche d'azione. I vecchi condizionamenti lungi dall'essere sostituiti o trasformati resistono generando un processo di frammentazione della struttura: i tentativi volti ad iniettare logiche manageriali e/o neo professionali in un tipico sistema burocratico-professionale creano un quadro complesso dove i condizionamenti precedenti continuano ad esercitare le loro pressioni, ma in modo non più esclusivo e pienamente legittimato come in passato. È proprio durante questo processo di riforma, che si introduce in ambito scolastico la richiesta di dirigenti scolastici "competenti», forse proprio per assorbire quella quota di ambiguità e incertezza del sistema. Il dirigente della scuola, infatti, da un lato viene sollecitato ad assumere una competenza manageriale, ma allo stesso tempo non ha una gestione piena delle ri- 
sorse strategiche, in primo luogo quelle umane, così come non prende rilevanti decisioni autonome in merito alle scelte formative che possono distinguere la propria offerta formativa. La pressione neoprofessionalista, d'altra parte, reagisce, sollecitando una figura di dirigente scolastico competente ma con una centratura prevalente sulla leadership educativa e sull'empowerment professionale, anche attraverso la riscoperta dei codici deontologici (Serpieri, 2002).

\section{L'emergenza delle "competenze trasformative» (TR)}

In questo paragrafo analizzeremo lo sviluppo ed il ruolo delle competenze emergenti dei leader educativi in carne ed ossa alle prese con i reali contesti di interazione, facendo ricorso allo studio di caso presentato precedentemente.

\section{Il tempo $T_{1}$ : la pre-autonomia}

Il preside, un ingegnere che esercita tale ruolo da più di quindici anni, dal 1993 dirige un istituto tecnico industriale. Dichiara di aver scelto l'insegnamento già in prospettiva del concorso come preside, si riteneva, infatti, più adatto a tale ruolo e lo ha sempre associato ad un'attività gratificante e dinamica che gli consentisse l'autonomia necessaria per influire in modo ampio ed efficace sulle attività formative ed educative. Fin dall'inizio della sua carriera il preside sentiva il desiderio di poter incidere significativamente sul miglioramento complessivo delle attività che concorrono al successo formativo, non solo quelle «in classe». Dopo 4 anni di insegnamento, infatti, inizia ad esercitare il ruolo di vicario, affrontando varie e numerose esperienze che valuta come molto gratificanti e significative per la sua formazione professionale. Si può ritenere che la scelta del ruolo, quindi, sia legata ad una motivazione intrinseca che ha spinto l'ingegnereinsegnante a vedere nella sua professione un lavoro in cui potersi pienamente realizzare.

La situazione iniziale dell'istituto assegnatoli in qualità di preside viene così descritta:

Condizionamenti struttural-culturali: si tratta di un istituto in provincia di $\mathrm{Na}$ poli, con circa 800 studenti, un tasso di dispersione molto superiore alla media regionale e un tessuto economico abbastanza povero. Al gravoso problema della dispersione, particolarmente critica nel biennio, si aggiunge anche un problema di pluri-ripetenza e di assenteismo. I ragazzi sono descritti come particolarmente demotivati, distratti e con scarso rendimento e le famiglie risultano completamente disinteressate. La struttura organizzativa dell'istituto è molto "piatta», oltre agli insegnanti ed al preside, l'unico ruolo formale più significativo è quello del vice preside che di fatto esercita una funzione di supporto e sostegno per tutte le attività dell'istituto. La cultura è spiccatamente di tipo burocratico-professionale ed, infatti, l'individualismo professionale degli insegnanti è molto accentuato. Nonostante mille difficoltà, gli insegnanti sono amati dai ragazzi e, con un duro lavoro in classe, riescono a fronteggiare i numerosi problemi di un contesto «a rischio».

Il preside inizia a riflettere sulla situazione dell'istituto («conversazioni inter- 
iori») e, in sintonia con gli insegnanti, individua come problema principale l'elevato tasso di dispersione. Crede, anche in base alle sue trascorse esperienze di insegnante e vicario, che sia necessario affrontarlo non solo attraverso le classiche misure di contrasto alle dispersione, ma agendo anche sulle aspettative negative che gli studenti hanno rispetto alla futura spendibilità, a livello lavorativo, del diploma conseguito nell'istituto. Ritiene, quindi, che si debbano necessariamente adottare misure «indirette» di contrasto alla dispersione che richiedono una diversa e più globale politica che coinvolge tutto l'istituto e soprattutto gli insegnanti. Il preside afferma che gli insegnanti, se spronati e incitati, sono: «il motore del cambiamento, sono fonti inesauribile di energie che aspettano solo di essere indirizzati verso obiettivi nuovi che possono migliorare il successo formativo». Il preside, quindi, in questi anni non solo supporta alcuni insegnanti nell'ottenimento di maggiori finanziamenti per i progetti di contrasto alla dispersione (molti dei quali riuscirà a finanziarli anche attraverso i fondi europei), ma definisce altri due obiettivi:

- azioni per migliorare la preparazione tecnico-professionale degli alunni;

- azioni di orientamento e supporto all'inserimento lavorativo e professionale.

Attraverso un rapporto di stretta collaborazione con un piccolo gruppo di insegnanti, ingegneri che già conosceva durante il percorso universitario, e dopo numerosi tentativi, la maggior parte dei quali si rilevano fallimentari, il preside ottiene, con un fondo europeo gestito dalla Regione, due laboratori per il controllo qualità, ritenuti all'avanguardia nel panorama locale, e un nuovo laboratorio informatico. In modo autonomo, inoltre, stringe relazioni con il tessuto imprenditoriale locale, e ben presto i laboratori vengono utilizzati anche da alcune aziende che si rendono disponibili per attività di tirocinio e stage post-diploma a beneficio degli alunni dell'istituto.

Alla fine del tempo $T_{1}$ possiamo notare numerosi cambiamenti.

Condizionamenti struttural-culturali: il numero delle iscrizioni cresce e la struttura organizzativa diviene molto più complessa. Con l'aumento dei progetti (a quelli per la lotta alla dispersione si aggiungono le attività di organizzazione e gestione di stage e tirocini) e dei laboratori, alcuni insegnanti acquisiscono nuovi ruoli (responsabili o referenti di progetto e laboratorio) che danno loro maggiore responsabilità e autonomia. Anche la figura di collaboratore del preside acquisisce sempre più rilevanza, sono proprio i suoi colleghi ingegneri ad acquisire e a mantenere tale incarico durante questi anni. Un numero sempre maggiore di insegnanti partecipa a vario titolo ai progetti e, attraverso queste esperienze, maturano una discreta competenza nel lavoro di gruppo e per progetti. Anche le attività laboratoriali costituiscono importanti occasioni $\mathrm{di}$ confronto che impegnano molti docenti in attività di innovazione e sperimentazione didattica. La cultura di tipo burocratico-professionale sopravvive, ma (con varie e a volte significative differenze) la partecipazione alle attività «extra aula» (laboratori, progetti, attività extra curriculari) coinvolge più del $50 \%$ degli insegnanti, i quali sono abbastanza favorevoli alle politiche del preside. Esiste però un certo mal- 
contento che non viene espresso in modo manifesto: alcuni insegnanti si sentono esclusi dal processo di cambiamento e maturano un certo disagio nei confronti di un preside visto come inaccessibile e attorniato da un gruppo di stretti collaboratori, che non lascia spazio ad altri colleghi. Ciò nonostante i docenti sono orgogliosi di lavorare in un istituto che attrae un numero sempre più consistente di alunni e gode di una reputazione molto elevata.

In riferimento al preside, le esperienze professionali gli hanno permesso di acquisire e sedimentare un set di "competenze emergenti»:

Le «competenze emergenti» al termine del tempo $T_{1}$ :

- si prefigge obiettivi concreti e non si scoraggia a fronte di esiti non sempre del tutto soddisfacenti;

- cura molto i rapporti con le istituzioni esterne (soprattutto per reperire maggiori fonti di finanziamento per le numerose attività che l'istituto organizza) ed, inoltre, coltiva personalmente una rete con la piccola realtà imprenditoriale locale per agevolare l'inserimento nel mercato del lavoro degli alunni;

- assume uno stile di leadership informale ed ha capacità di empowerment, ma limitata a singoli collaboratori di vecchia conoscenza con i quali ha stabilito stretti rapporti di fiducia e con cui riesce a lavorare in team scambiandosi ruoli e competenze con notevole fluidità.

- con l'aumento degli insegnanti coinvolti in incarichi aggiuntivi, acquisisce uno stile prevalentemente manageriale e attua un ampio processo di delega spingendo l'organizzazione verso un sempre maggiore processo di razionalizzazione e formalizzazione delle attività.

Tali competenze, quali proprietà emergenti costituiranno una parte dei vincoli e delle risorse a sua disposizione dal tempo $\mathrm{T}_{2}$ al $\mathrm{T}_{3}$.

\section{Il tempo $T_{2}-T_{3}$ : la riforma}

Siamo giunti nell'anno scolastico 2000/2001, il neo dirigente dichiara di aver accolto la Riforma dell'Autonomia scolastica con molto entusiasmo, ma quando descrive i cambiamenti pratici e concreti relativi all'esercizio del suo ruolo, ben presto afferma che in realtà il suo modo di impersonificare tale ruolo non è molto diverso. Il dirigente stibilise una proficua e fattiva collaborazione con le nuove figure di staff (f.o.) ${ }^{8}$. Il dirigente, inoltre, dichiara di voler continuare a migliorare la collocazione lavorativa dei suoi alunni incorriggiato dai buoni risultati ottenuti. Anche se gli insegnanti richiedono nuove risorse per i progetti contro ledispersione, il dirigente, pur riconoscendo la legittimità delle loro istanze, decide di effettuare una doppia strategia:

- da un lato, vuole migliorare l'employability degli alunni attivando bilanci di competenze (Alby \& More, 2004) individuali per l'orientamento e stage in imprese più competitive e moderne dislocate in aree più lontane, ed, inoltre, vuole diffondere le opportunità formative di tipo universitario; 
- dall'altro lato, intende valorizzare soprattutto verso l'esterno (le famiglie dei nuovi possibili studenti) le ricadute positive della sua politica; le sue azioni ed i relativi risultati hanno maggiore evidenza probatoria rispetto al tempo $T_{1}$, poiché in quello da $T_{2}$ a $T_{3}$ deve render conto (processi di etero ed autovalutazione) dell'efficacia delle iniziative. In tale attività coinvolge le f.o.

Paradossalmente è con l'inserimento delle figure di staff che sorgono nuovi problemi. I docenti iniziano a lamentarsi esplicitamente perchè le f.o. sembrano aver preso il ruolo del dirigente che, non ascolta le loro proposte e sembra orientato solo verso la costruzione di una scuola di qualità. Tra il dirigente e la f.o. che dovrebbe interessarsi dei rapporti con gli enti esterni, inoltre, sorgono numerosi problemi. L'insegnante possiede un discreto potenziale, ma non ha maturato esperienze e soprattutto non ha mai collaborato con la presidenza. Il dirigente, che ha sempre curato personalmente i rapporti con le aziende e ha già da tempo affidato ai suoi collaboratori la gestione dei fondi europei, ritiene che la funzione obiettivo debba sostenere il lavoro dei colleghi (le altre f.o.) e non vuole integrarla nell'attività formalmente attribuita.

Dopo solo un anno, la situazioni può essere così descritta.

Condizionamenti struttural-culturali: il numero delle iscrizioni cresce e l'istituto ospita 1150 alunni; la struttura segue il trend della progressiva complessificazione con la nascita di nuovi ruoli. Le f.o. razionalizzano e formalizzano i compiti e i ruoli (stesura di organigrammi, diagrammi di flusso, gant, ecc.). Il clima della scuola è più teso e nel collegio dei docenti alcuni manifestano aspre rivendicazioni sulle modalità di gestione del fondo di istituto. È possibile rintracciare tre diversi condizionamenti che si possono ricondurre alle nuove spinte che il sistema introduce con la riforma:

- la maggioranza dei docenti, supportati anche da un piccolo gruppo di genitori, appoggiano il dirigente nel privilegiare le attività di employability rispetto a quelle dirette al sostegno dei drop out, ritenendo il recupero, esclusivamente formale, inutile e, spesso, dannoso. La scelta si è rilevata di successo, infatti, il numero di iscrizioni è altissimo. Questo gruppo di insegnanti può essere ritenuto espressione del condizionamento al managerialismo visto il loro interesse per una scuola di «qualità» che sia concorrenziale nei confronti degli altri istituti limitrofi;

- un altro gruppo, in opposizione al precedente, non vuole avallare la scelta meritocratica e esprime perplessità verso stage e tirocini che richiedono alle famiglie sacrifici economici ulteriori e non offrono garanzie. In alternativa, richiedono il massimo investimento sulle misure di lotta alla dispersione. Questo gruppo può essere visto come espressione delle correnti neo-professionali, infatti, privilegiano l'equità sociale e sono molto contrari verso le nuove modalità di razionalizzazione, valutazione e controllo delle attività didattiche;

- un terzo gruppo di insegnanti resta abbastanza in disparte e non prende parte alla polemica, anzi dichiara che sembra ingiusto gravarsi di compiti che non sono di loro competenza e senza godere di alcun incentivo, mentre le altre is- 
tituzioni competenti in materia risultano «latitanti». Il costante richiamo all'aderenza a quanto previsto dal contratto e le numerose rivendicazioni sindacali fanno vedere questo gruppo come manifestazione del condizionamento al burocratismo.

La sintesi dei risultati di questi anni di attività è comunque più che soddisfacente:

- il numero delle iscrizioni è notevolmente aumentato e l'istituto ha ottenuto una nuova e più efficiente sede;

- il tasso di dispersione continua ad essere molto basso e l'istituto viene sempre apprezzato e riconosciuto come un fiore all'occhiello della realtà locale;

- il bacino di nuove aziende non è migliorato granché;

- il numero delle iscrizioni all'università (soprattutto ingegneria) è aumentato grazie alle varie iniziative con il politecnico;

- solo per un anno scolastico sono stati attivati i bilanci di competenze per gli alunni dell'ultimo anno;

- è stato attivato un corso biennale in collaborazione con i dipartimento di ingegneria dell'università Federico II.

L'ingegnere non ha risolto i vari problemi e non ha trasformato le proprie competenze, tuttavia ritiene che il nuovo ruolo è ancora più stimolante e questo viene confermato dal fatto che non accetta di lasciare la scuola per assumere un incarico presso il ministero e che, nonostante le numerose difficoltà, si prefigge sempre nuovi obiettivi di miglioramento.

\section{La spirale delle "competenze emergenti» (TR)}

L'acquisizione delle competenze è rappresentata attraverso un processo a spirale (Fig. 4) per sottolineare come il leader educativo, da un lato, interpreta un ruolo e, quindi, interagisce in un reale contesto e, dall'altro, ritorna continuamente sui suoi vari impegni, organizza le priorità, filtra, valuta e riflette sulle emozioni relative ai successi o agli insuccessi che ottiene. Nel ricostruire tale percorso, quindi, si sono rintracciati i ruoli emblematici che la medesima persona interpreta durante la sua vita professionale. Il leader educativo da noi studiato è prima preside e poi dirigente, ma, agli inizi della carriera, è anche un insegnante e un vicario. $\grave{E}$, infatti, con l'esperienza da insegnante che si sente frustrato per il limitato impatto che riesce ad avere sul successo formativo dei suoi alunni e, quindi, accetta ed interpreta con entusiasmo quello di vicario. Inizia così una nuova attività e il suo percorso si arricchisce di esperienze che anticiperanno, per molti versi, quelle successive. Nel periodo $\mathrm{T}_{1}$, divenuto preside, l'ingegnere si pone come principale obiettivo quello di innescare, nell'istituto togli, un processo di radicale cambiamento che, di fatto, coinvolgerà la maggiore parte del corpo docente. Dopo pochi anni, e non senza difficoltà, raggiunge con successo i suoi obiettivi creando, da un contesto passivo e refrattario, isomorfico all'assetto sistemico, un istituto all'avanguardia. L'attore dimostra di avere acquisito quelle che si sono definite "competenze trasformative», ovvero la capacità di 
rompere le routine e di innescare un processo di cambiamento non reversibile, che dimostra il potere dell'attore e delle sue competenze rispetto ai contesti sociali e che contrastaefficacemente i condizionamenti burocratico-professionali del sistema.

Nel periodo successivo $\left(\mathrm{T}_{2}-\mathrm{T}_{3}\right)$, il percorso viene movimentato da nuovi condizionamenti esterni innescati con la riforma dell'Autonomia. Tali cambiamenti, per certi versi, lo appoggiano poiché, divenuto dirigente, si trova ad operare potenzialmente con più risorse e maggiore autonomia, ma le nuove sisteme richiede una crescente quota e/o una diversa qualità di competenze necessarie per gestire e coordinare istanze che spesso sono totalmente divergenti (managerialismo versus burocratismo, professionalismo versus neo-professionalismo. Il dirigente dovrebbe, quindi, acquisire nuove competenze; ma questo è un esito non scontato ed imprevedibile, in quanto sensibile alle proprietà emergenti delle competenze stesse e un sistema scolastico in trasformazione.

Nel suo percorso a spirale, il dirigente si trova in un punto molto delicato: egli è entusiasta dei cambiamenti (l'autonomia finanziaria, didattica ed organizzativa, la dirigenza stessa, l'introduzione delle funzioni obiettivo, ecc.), ma, dopo più di venti anni servizio tra vicariato e presidenza, ha acquisito una quota di "competenze emergenti», durature e relativamente indipendenti. Il dirigente riflette (conversazioni interiori) sulla nuova situazione e, ad esempio, comprende la necessità di delegare alle f.o. molte attività, ma non riesce a delegare la gestione delle relazioni esterne. Egli ha allacciato relazioni personalistiche soprattutto con i rappresentanti delle aziende e non intende coinvolgere minimamente la f.o. formalmente incaricata di questo compito. Pur essendo consapevole dell'incresciosa situazione, non trova una soluzione perché sostanzialmente le sue «competenze emergenti» gli impediscono di passare dal ruolo di "trasformatore» e "promotore» a quello di «mediatore». Il dirigente è ormai «competente» nell'effettuare i progetti di innovazione in modo autonomo (si confronta solo con i collaboratori ed ora le f.o. che lo riconoscono come leader indiscusso) e nel coinvolgere gli insegnanti senza che questi possano negoziare i suoi propositi. Ora che alcuni insegnanti manifestano apertamente il loro dissenso, il dirigente ha notevoli difficoltà a dialogare con portatori di istanze contrapposte e sceglie o di evitare l'incontro-scontro o di delegarlo alle f. o. La sua maggior gratificazione personale è, infatti, sentirsi promotore e ideatore del cambiamento, ruolo che non vuole condividere con facilità, soprattutto con chi non conosce bene e che non vede ancora come un suo sostenitore:

$i$ miei collaboratori fanno tutto loro ... sono fantastici, io dico cosa mi voglio inventare e loro subito partono, con loro lavoro da anni ... le f.o. ... è stata una conferma di quattro insegnanti che gì̀ mi seguivano, ... dico vorrei cambiare questo e loro mi seguono ... ora ho delegato tantissimo perché so che loro mi seguono, sono attente, preparate. ... Io mi sono inventato da solo un istituto nuovo, ma senza di loro [gli insegnanti] non l'avrei mai costruito... il dirigente ha il compito di guidare gli insegnanti verso obiettivi di miglioramento molto ampi, obiet- 
tivi a lungo periodo, che loro nella classe non vedono anzi se li vedessero si spaventerebbero... [in riferimento ai problemi con la f.o.] è difficile seguirmi quando sto ideando, quando ci sono cose che devo inventarmi giorno per giorno con persone che si fidano di me e che accettano di lavorare perché sanno che ci sono io. Cosa posso delegare se non so bene cosa farò? e se non so cosa è capace di fare lui quando a me viene unidea? $X$ deve aiutare gli altri nei compiti che io posso delegare.

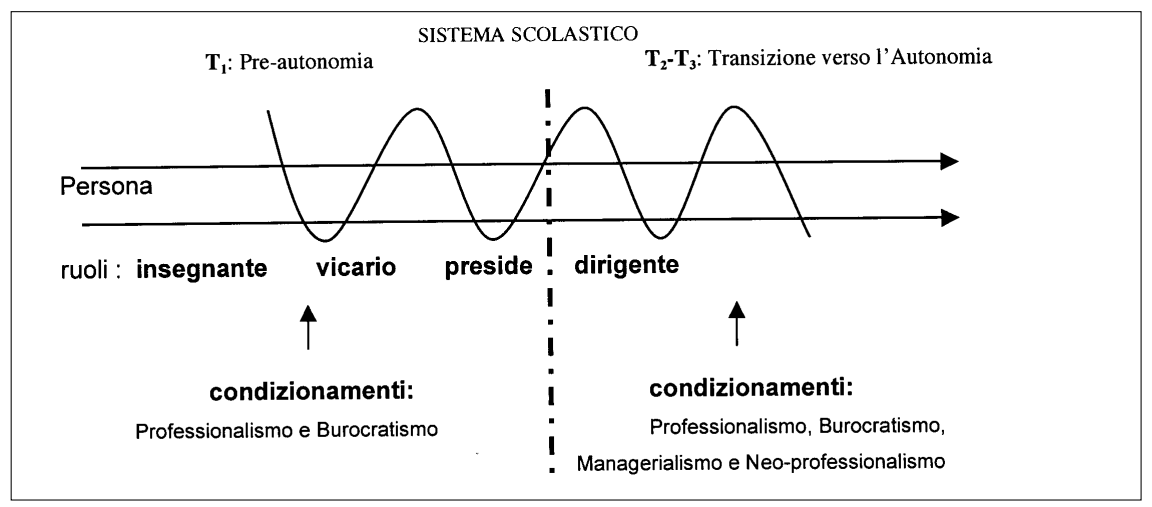

Fig. 4 Spirale delle competenze emergenti di un dirigente scolastico

\section{Leader, politiche di riforma e "competenze emergent $i$ »: una riflessione conclusiva (TR)}

L'obiettivo del presente lavoro è fornire un contributo all'analisi dell'agire professionale e, quindi, all'analisi delle scelte che un individuo effettua in qualità di reggitore di uno specifico ruolo lavorativo. L'attore è stato concettualizzato come un soggetto in possesso di poteri e proprietà autonome (proprietà emergenti) che lo rendono capace di riflettere (conversazioni interiori) e di agire sul contesto in cui vive e anche di trasformarlo. Tale potere di riflessione e azione è condizionato dal livello di competenza raggiunto dai soggetti. Il concetto di competenza proposto ci permette di rivedere l'azione in una prospettiva stratificata (abbandonando letture dell'agire di tipo conflattivo, come proposto in Romano \& Serpieri, 2003), ricostruendo la vita professionale e lavorativa in una più ampia configurazione. I ruoli sociali sono l'espressione dei condizionamenti socioculturali e sono interpretati ed agiti alla luce di differenti vissuti emotivi, cognitivi ed esperenziali. Cercando di realizzare gli obiettivi radicati negli strati più profondi della propria identità, infatti, l'attore riveste un ruolo e subisce (in positivo o in negativo) i condizionamenti che gratificano o frustrano le sue aspettative. Tali esperienze concrete costituiscono un costante e ripetuto banco di prova e permettono al soggetto di rinforzare o modificare le sue competenze. 
Attraverso la storia narrata, si è analizzata la lenta costruzione delle competenze di un leader educativo. Un processo che avviene in un ambiente soggetto a diversi condizionamenti ed a più logiche di azione, ivi compresa quella dell'attore stesso con la sua storia professionale; si crea un processo a spirale che vede il leader attraversare vari ruoli e sedimentare diverse competenze, di cui in qualche misura non è egli stesso fino in fondo consapevole e sovrano. Si tratta, quindi, di un processo dagli esiti aperti la cui lettura deve tenere necessariamente conto del dualismo analitico (relazione attore e struttura-cultura) e della prospettiva temporale (morfogenesi ed emergenza). In Italia, ad esempio, il sistema nel tempo $\mathrm{T}_{1}$, se da un lato spingeva verso un ruolo burocratico ed amministrativo, dall'altro, permetteva agli attori, una volta soddisfatte le richieste formali, di realizzare una quota di comportamenti discrezionali la cui accountability si muoveva su piani estremamente laschi. L'attore può anche esercitare il suo potere verso il contesto e, come nel caso esaminato, il preside sceglie di ricavarsi alcuni spazi di autonomia ed attuare un processo di trasformazione del proprio istituto. Nel periodo $T_{2}-T_{3}$ inizia un processo di cambiamento che risente non solo dei mutamenti del sistema (una riforma caratterizzata da numerosi margini di incertezza), ma anche dei vari contesti locali, le cui configurazioni sono frutto dell'azione precedente dei protagonisti (i leader educativi innanzitutto). Analizzare le competenze dei dirigenti costituisce, un criterio di lettura dei possibili esiti di prassi didecentuamento e di governance poiché i leader, che hanno il delicato compito di trasformare $\mathrm{i}$ contesti locali favorendo la riappropriazione della loro autonomia, rivestono un ruolo centrale e strategico. L'andamento di una riforma se, per molti versi, è sconosciuto, non è completamente indeterminato poiché, attraverso l'analisi delle competenze delle figure decisive (come i leader), possiamo comprendere l'evoluzione di processi che si basano sull'attivazione dei contesti.

La competenza come frutto dell'interazione tra elementi radicati allo strato dell'identità personale e i condizionamenti del contesto, quindi, costituisce un fenomeno ricco di interesse, ma non ancora pienamente esplorato. La recente letteratura sulle competenze ci ha, infatti, permesso di superare una lettura dell'attore come un mero epifenomeno delle determinanti sociali che al più aveva l'esclusivo potere di «resistere al cambiamento». L'attore competente che agisce in spazi di interazione sempre più liberi di autodeterminazione e autodecisione, non ci deve, però, far cadere nell'errore opposto e speculare, consegnandoci un attore senza storia, senza società e soprattutto senza identità.

\section{Note}

1 Questo articolo rappresenta una rielaborazione ed uno sviluppo interpretativo di un precedete lavoro degli autori (Romano \& Serpieri, 2006).

2 Emblematico in tale direzione il cambiamento del titolo della rivista Educational Management \& Administration in Educational Management, Administration \& Leadership nel 2004 (si ringrazia Giovanna Barzanò per questa sottolineatura). 
3 Mentre le micropolitiche di leadership di Blase evidenziano espliciti richiami ai lavori di Ball, per contro, vi è una sostanziale mancanza di riferimenti alla letteratura sulla leadership trasformazionale.

4 Il concetto di competenza si trova al centro di varie prospettive: dalla teoria sociologica (Giddens, 1984 e, per certi versi, Sen, 1994) agli studi organizzativi (Gherardi \& Nicolini, 2004), senza trascurare gli ambiti più squisitamente applicativi, come la gestione del personale (Spencer \& Spencer, 1993). Nell'economia del presente lavoro, definiamo la competenza come la capacità di vivere in un mondo moderno, ovvero quell'insieme di capacità che permettono all'individuo di agire riflessivamente e di tenere il timone della propria esistenza sia nella sfera privata sia nei contesti sociali e lavorativi, dove le trasformazioni della struttura spingono l'individuo a farsi sempre più carico dell'incertezza e del rischio. È sembrato, quindi, il concetto più idoneo per intercettare ed analizzare i cambiamenti e le trasformazioni che investono numerose figure professionali che affrontano forse per la prima volta impegni e responsabilità del tutto nuovi.

5 La Archer ha una visione stratificata delle società e degli individui. È necessario quindi riconoscere i diversi strati attraverso le "proprietà emergenti" sia della struttura e della cultura, con le loro influenze e vincoli, sia degli individui con la loro relativa autonomia poiché il reciproco costituirsi tra azione e struttura non elimina la necessità/possibilità di analizzarli in modo distinto (dualismo analitico). Anche l'individuo ha quindi proprietà emergenti ovvero strati (sé, persona, agente e attore) che hanno caratteristiche e poteri che li rendono distinti gli uni dagli altri analiticamente: autonomia relativa, priorità temporale e efficacia casuale. In particolare esistono le tre seguenti emergenze: attore, agente e persona. Ciò consente di vedere come l'azione (livello dell'attore), pur avendo sempre margini di libertà, è condizionata dalla struttura sociale (livello dell'agente), ma anche dalla struttura psicologica interna agli individui (livello della persona). Il condizionamento della struttura, in altri termini, posiziona gli esseri umani e li costituisce nello strato degli "agenti», che si raggruppano in quanto condividono «le stesse possibilità di vita». I condizionamenti, quindi, non determinano l'azione che resta libera pur subendo, in positivo o in negativo, $\mathrm{i}$ costi ed i benefici dettati dal posizionamento nella struttura.

6 La Bei - Behavioural Event Interview - è un' intervista "comportamentale» semi-strutturata in cui si chiede agli intervistati di raccontare alcuni episodi realmente accaduti durante la propria vita lavorativa, in genere tre che hanno dato vita a performance di successo e tre che hanno vita a performance di insuccesso. L'intervista ha l'obiettivo di ricostruire attentamente tutto quello che è accaduto durante l'episodio, cercando di identificare le numerose variabili in gioco (contesto organizzativo, persone coinvolte, risorse a disposizione), tutte le azioni che ha svolto il protagonista e di indagare gli schemi cognitivi, le intenzioni e le motivazioni che hanno guidato l'intervistato (Spencer \& Spencer, 1993). Tale tecnica è stata utilizzata anche per indagare in profondità le competenze delle figure di staff (Esposito, Romano, \& Serpieri, 2000).

7 Si presenta anche nel caso italiano la comparsa di diverse dimensioni di questo movimento neoprofessionale come avvenuto anche in altri sistemi educativi. Alcune organizzazioni professional-sindacali, ad es., arrivano sino a rivendicare una modalità elettiva per i dirigenti scolastici, così come avviene per le università. Un altro indicatore significativo può essere rintracciato nella presentazione da parte di un'associazione di categoria particolarmente accreditata di un codice etico per i dirigenti scolastici; così come le varie forme di protesta per le nuove, sia pure sperimentali, metodologie di valutazione.

8 Con la riforma, nel 1999, vengono introdotte le funzioni obiettivo, insegnanti scelti dal collegio dei docenti che si ripartiscono i compiti in base alle aree tematiche a loro attribuite: la gestione del piano di offerta formativa, il sostegno al lavoro dei docenti, gli interventi e servizi per gli studenti, le relazioni con gli enti esterni. 


\section{Riferimenti bibliografici}

Alby, F., Mora, F. (2004). Il bilancio di competenze. Roma: Carocci

Archer, M. S. (1997). La morfogenesi della società. Milano: Angeli. (Opera originale pubblicata nel 1995)

Archer, M. S. (2000). Being Human: The Problem of Agency. Cambridge: Cambridge University Press.

Archer, M. S. (2003/2006). Structure, Agency and Internal Conversation (trad. it. La conversazione interiore. Trento: Erikson).Cambridge: Cambridge University Press.

Bacharach, S. B. \& Mundell, B. (1993). Organizational Politics in schools: Micro, macro and logics of actions. Educational Administration Quarterly, 29, 423-452.

Bacharach, S. B. \& Mundell, B. (1995). Images of Schools. Structures and Roles in Organizational Behavior. Thousands Oaks, Ca.: Corwin Press.

Ball, S. J. (1987). The Micro-Politics of the school. Towards a theory of school organizations. London: Methuen.

Ball, S. J. (1994). Education Reform. Buckingham: Open University Press.

Ball, S. J. (2006). Education Policy and Social Class. London: Routledge.

Battistelli, F. (A cura di). (2002). La cultura delle amministrazioni pubbliche fra retorica e innovazione. Milano: Angeli.

Barzanò, G. (in stampa). Leadership ed educazione: idee e proposte. Roma: Monolite.

Battistelli, F. (A cura di). (2002). La cultura delle amministrazioni pubbliche fra retorica e innovazione. Milano: Angeli.

Benadusi, L. (A cura di). (1989). La non decisione politica. Firenze: La Nuova Italia.

Benadusi, L. (2002). La «logica competenza» nella scuola . In L. Benadusi, G. Di Francesco (A cura di). Formare per competenze. Un sentiero di innovazione tra scuola e formazione professionale ( $p$ p. 7-72) : Napoli: Tecnodid.

Benadusi, L. \& Landri, P. (2002). Verso la governance. L'eclissi della burocrazia scolastica e la costruzione del sistema dell' istruzione e della formazione. Economia \& Lavoro, 1, 163-182.

Benadusi, L. \& Serpieri, R. (A cura di). (2000a). Organizzare la scuola dell' autonomia. Roma: Carocci.

Benadusi, L. \& Serpieri, R. (2000b). La scuola dell'autonomia: problemi di organizzazione e di valutazione. In L. Benadusi \& R. Serpieri (A cura di), Organizzare la scuola dell' autonomia (pp.11-36). Roma: Carocci.

Biggiero L. (2000). Il sistema scolastico italiano: un approccio post-fordista. In L. Benadusi \& R. Serpieri (A cura di), Organizzare la scuola dell' autonomia (pp.157-196). Roma: Carocci.

Blase, J. \& Anderson, G. L. (1995). The micropolitics of educational leadership. London: Cassel.

Bryman, A. (1992). Charima \& Leadership. London: Sage.

Bullock, A. \& Thomas, H. (1997). Schools at the Centre? London: Routledge.

D’Albergo, E. \& Vaselli P. (1997). Un'amministrazione imprenditoriale? Roma: Seam.

Day, C., Harris, A., Hadfield, M., Tolley, H. \& Beresford, J. (2000). Leading Schools in Times of Change. Buckingham: Open University Press.

Dimmock, C. \& Walker, A. (2005). Educational Leadership. Culture and Diversità. London: Sage.

Esposito, S., Romano, I. \& Serpieri, R. (2000). Funzioni obiettivo e innovazione delle competenze nella scuola dell'autonomia. Scuola democratica, n. 4, 37-58.

Fisher, L., Fisher, M. G. \& Masuelli, M. (2002). I dirigenti nella scuola dell'autonomia. Bologna: Il Mulino.

Gherardi S. \& Nicolini D. (2004). Apprendere e conoscere nelle organizzazioni. Roma: Carocci.

Giddens, A. (1990). La costituzione della società. Milano: Edizioni di Comunità. (Opera originale pubblicata nel 1984)

Gronn, P. \& Ribbins, P. (1996). Leaders in Context: Postpositivist Approaches to Understanding Educational Leadership. Educational Administration Quarterly, vol. 32, n. 3, 452-473. 
Gronn, P. (2000). Distributed Properties: A new Architecture for leadership in Educational Management \& Administrtion, 28, n. 3, 317-338.

Hargreaves, A. \& Fink, D. ( 2003). The Seven Principles of Sustainable Leadership. Educational Leadership, 61, 8-13.

Helsby, G. (2002). Come cambia il lavoro degli insegnanti, Firenze: Libri liberi. (Opera originale pubblicata nel 1999)

Hodgkinson, C. (1991). Educational Leadership: The Moral Art. Albany: SUNY Press.

Leithwood, K., Chapman, J., Corson D., Halinger P. \& Hart A. (Eds). (1996). International handbook of educational leadership and administration. Dordrecht: Kluwer Academic Publishers.

Leithwood, K., Jantzi, D. \& Steinbach, R. (1999). Changing Leadership for Changing Times. Buckingham: Open University Press.

Levacic, R., Glover, D., Bennett, N. \& Crawford, M. (1999). Modern Headship for the Rationally Managed School: Combining Cerebral and Insightful Approaches. In T. Bush, L. Bell, R. Bolam, R. Glatter \& P. Ribbins (Eds.). Educational Management: Redefining Theory, Policy and Practice (pp.15-28). London: Paul Chapman Publishing Ltd.

Mintzberg, H. (1985). La progettazione dell'organizzazione aziendale. Bologna: Il Mulino.

Ribolzi, L. (1997). Il sistema ingessato: autonomia, scelta e qualità nella scuola italiana. Brescia: La Scuola.

Romano, I. \& Serpieri, R. (2003). Per una concezione stratificata dell'»attore competente». Sociologia del Lavoro, n. 92, 134-150.

Romano I. \& Serpieri R. (2004). Le competenze della leadership scolastica: la spirale delle proprietà emergenti. In L. Benadusi \& F. Consoli (A cura di), La governace della scuola (pp.147-183). Bologna: Il Mulino.

Romano, I. \& Serpieri, R. (2006). Dirigenti e costruzione della leadership scolastica. In M. Colombo, G. Giovannini \& P. Ladri (A cura di), Sociologia delle politiche e dei processi formativi (pp.301-322). Milano: Edizioni Guerini.

Sadler, P. (2000). Leadership and Organizational Learning. In Handbook of Organizational Learning and Knowledge (pp.415-427). Oxford: Oxford University Press.

Selznick, P. (1976). La leadership nelle organizzazioni: Milano: Angeli. (Opera originale pubblicata nel 1957)

Sen, A. (1994). La disuguaglianza. Un riesame critico. Bologna: Il Mulino.

Sergiovanni, T. J. (1992). Moral Leadership: Getting to the Heart of School Leadership. San Francisco: Jossey-Bass.

Serpieri, R. (1999). Tra identità individuale e sociale: il dirigente scolastico come persona e come attore. Scuola democratica, n. 3-4, 89-104.

Serpieri, R. (2002). Leadership senza gerarchia. Riflessioni sul management scolastico. Napoli: Liguori Editore.

Shedd, J. B. \& Bacharach, S. B. (1991). Tangled Hierarchies: Teachers as Professionals and the management of schools. San Francisco: Jossey-Bass.

Silverman, D. (200/2002). Doing qualitative research. A practical guide (trad. it. Como fare Ricerca Qualitativa. Roma: Carocci). London: Sage Publications.

Sims, H. P. \& Lorenzi, P. (1992). The New Leadership Paradigm. Newbury Park: Sage.

Spencer, L. M. \& Spencer, S. M. (1995). Competenza nel lavoro, Milano: Angeli. (Opera originale pubblicata nel 1993)

Telford, H. (1996). Transforming schools throguh collaborative leadership. London: Falmer Press.

Ventura, S. (1998). La politica scolastica. Bologna: Il Mulino.

Wallace, M. \& Weindling, D. (1999). Overview of a Group of Research Projects with Relevance to School Management. In T. Bush, L. Bell, R. Bolam, R. Glatter \& P. Ribbins (Eds.), Educational Management: Redefining Theory, Policy and Practice (pp. 206-217). London: Paul Chapman Publishing Ltd. 
Woods, P. A. (2005). Democratic Leadership in Education. London: Paul Chapman Publishing Ltd.

Parole chiave: leadership educativa, sistema scolastico italiano, competenza, conversazione, morfogenetica

\section{Leadership im italienischen Schulsystem: zwischen Institutionen und Kompetenzen}

\section{Zusammenfassung}

Ziel dieses Beitrags ist es, Schulführung in einer Perspektive der „wachsenden Kompetenz" (Nachhaltigkeit, Autonomie und Unabhängigkeit) zu betrachten, welche eine komplexe Beziehung zwischen Individuum und Kontext ausdrückt. Der pädagogische Leiter ist nichts anders als ein kompetentes Subjekt mit der Fähigkeit, sich selbst sowie die institutionellen Verbindlichkeiten und Ressourcen zu beobachten. Das vorgeschlagene Interpretationsschema wird in einen Fall angewendet: Es wird die Situation eines Leaders mit einer langen Berufserfahrung untersucht und zwar vor dem Hintergrund der mit hohen Ansprüchen an Schulführung gekoppelten Reform des italienischen Schulsystems. Die Leadershipkompetenzen gehen aus dieser komplexen Beziehung hervor. Sie bedingen sich gegenseitig ohne sich weder zu begründen (Ontologie der Praxis) noch sich zu determinieren (Reduktionismus), insofern sie nicht reduzierbar sind (gemäss dem kritischen Realismus archerian).

Schlagworte: schulische Leadership, italienisches Schulsystem, Morphogenese, Diskussion, Kompetenz 


\section{Le leadership éducatif dans le système scolaire italien: entre institutions et compétences}

\section{Résumé}

L'enjeu de cet article est de fournir une lecture du leadership éducatif à travers une perspective qui permette de voir celle-ci comme une "compétence émergente " (stable, autonome et indépendant) qui prend forme à partir de la relation complexe entre un individu et le contexte. Le leader éducatif n'est qu'un sujet compétent, en mesure de contrôler réflexivement (conversations internes) soimême (désirs, passions, projets) et les contraintes et les ressources institutionnelles. La clé de lecture proposée sera appliquée dans l'analyse d'un étude de cas : d'un côté, le contexte institutionnel (le système scolastique italien) bouleversé par une réforme qui exige des leaders l'acquisition de nouvelles compétences, de l'autre, un leader éducatif avec une longue carrière professionnelle derrière lui. La compétence de leadership apparaîtront de cette relation complexe entre des éléments qui se conditionnent entre eux sans se fondre ensemble (ontologie de la praxis) ni se déterminer (réductionisme), dans la mesure où il sont irréductibles (selon le réalisme critique archerian).

Mots clés: Leadership éducatif, système scolastique italien, compétence, conversation, morphogénétique

\section{The educational leadership in the Italian school system between institutions and competences}

\section{Summary}

The purpose of this article is to offer a reading of educational leadership as an "emerging competence» (durable, autonomous and independent), the result of the complex relationship between individual and context. The educational leader is nothing but a competent person, able to monitor reflexively (through inner conversation) herself (desires, passions, projects) and the institutional constraints and resources. The proposed framework will be applied to a case study: on one hand, an institutional context (the Italian school system) overrun by a reform process that pushes leaders to take on new competences (school management); on the other hand, an educational leader with a longstanding professional experience. The leadership competences will emerge from this complex relationship between elements that influence each other without melting into each other (ontology of praxis) nor determining each other (reductionism), in that they are not reductible (according to the Archerian critical realism).

Keywords: educational leadership, italian educational system, morphogenetic approach, internal conversation, competence 
T h e m a 